\title{
Galapaganus howdenae howdenae Lanteri, 1992 (Coleoptera, Curculionidae, Entiminae), ravageur potentiel du Palmier à huile (Elaeis guineensis Jacquin) en Équateur?
}

\author{
par Laurence Beaudoin-Ollivier ${ }^{1}$, Henri-Pierre AberLenc ${ }^{2}$ \\ \& Claude LouisE ${ }^{3}$ \\ ${ }^{1}$ Cirad-UPR Bioagresseurs, Campus de Baillarguet, F - 34398 Montpellier <laurence.ollivier@cirad.fr> \\ ${ }^{2}$ Cirad-CBGP, Campus de Baillarguet, 755 avenue du Campus Agropolis, F - 34988 Montferrier-sur-Lez cedex \\ $<$ henri-pierre.aberlenc@cirad.fr $>$ \\ ${ }^{3}$ Palmelit SAS, Parc Agropolis, 2214 boulevard de la Lironde, F - 34980 Montferrier-sur-Lez \\ <claude.louise@palmelit.com>
}

Résumé. - Des dégâts sur Palmier à huile occasionnés par Galapaganus howdenae ont été récemment observés dans une plantation de la province d'Esmeraldas en Équateur. Ce Charançon est polyphage et c'est son premier signalement comme ravageur potentiel du Palmier à huile.

Abstract. - Galapaganus howdenae howdenae Lanteri, 1992 (Coleoptera, Curculionidae, Entiminae), putative oil palm (Elaeis guineensis Jacquin) pest in Ecuador? Galapaganus howdenae was recently discovered in the Esmeraldas province in Ecuador damaging Oil Palm Trees in a plantation. This weevil is polyphagous and is recorded for the first time threatening Oil Palm Trees.

Keywords. - Weevil, Naupactini, Ecuador, defoliator, polyphagous, Pueraria phaseoloides, Oil Palm Tree.

Le genre Galapaganus Lanteri, 1992, inclut 15 espèces de Charançons présentes sur le continent sud-américain en Équateur et dans l'archipel des Galapagos, où 10 taxa sont endémiques. Les imagos consomment du feuillage et les larves se nourrissent de racines. La plupart des espèces semblent être polyphages (LANTERI, 1992 ; SEQUEIRA et al., 2000).

Galapaganus howdenae howdenae Lanteri, 1992 (fig. 1-3) est connu de l'archipel des Galapagos, sur l'île de Santa Cruz, depuis les zones cultivées de basse altitude jusqu'au Cerro Crocker (700-800 m) (LANTERI, 1992, 2004). La période estimée d'introduction accidentelle à Santa Cruz correspond à celle de la colonisation (1832-1959) (Мок et al., 2014). G. howdenae howdenae a été observé à Santa Cruz sur diverses plantes introduites, telles que Erythrina (SEQUEIRA et al., 2000), Psidium guajava L., Persea americana Mill. et sur une plante endémique des Galapagos, Tournefortia rufosericea Hook. f. (MoK et al. 2014). G. howdenae howdenae se rencontre en zone cultivée à Santa Cruz et en zone forestière sur le continent (SEQUEIRA et al., 2000). Ce Charançon est cité de la zone côtière de l'Équateur, dans les provinces de Guayas, Manabí et Los Ríos.

Galapaganus howdenae pecki Lanteri, 2004, est connu en Équateur de la province de Pichincha, à relativement haute altitude (250 à $1400 \mathrm{~m})$ (LANTERI, 2004).

G. howdenae howdenae est présent dans la province d'Esmeraldas, où il n'avait jamais posé de problème jusqu'à ce qu'il se mette brusquement à pulluler de septembre à novembre 2014, sur environ 60 ha d'une plantation de Palmier à huile Elaeis guineensis. Pour l'identification, 12 imagos des deux sexes ont été examinés.

Il n'avait jamais été signalé auparavant sur Palmier à huile, que ce soit par LEPESME (1947), Howard et al. (2001), Corley \& Tinker (2016) ou par Mariau (2001). Sur des palmiers âgés de moins de trois ans, il a occasionné de sérieux dégâts aux feuilles jeunes et moyennes en mangeant les folioles depuis le bord extérieur en direction de la nervure centrale (fig. 4-6). 

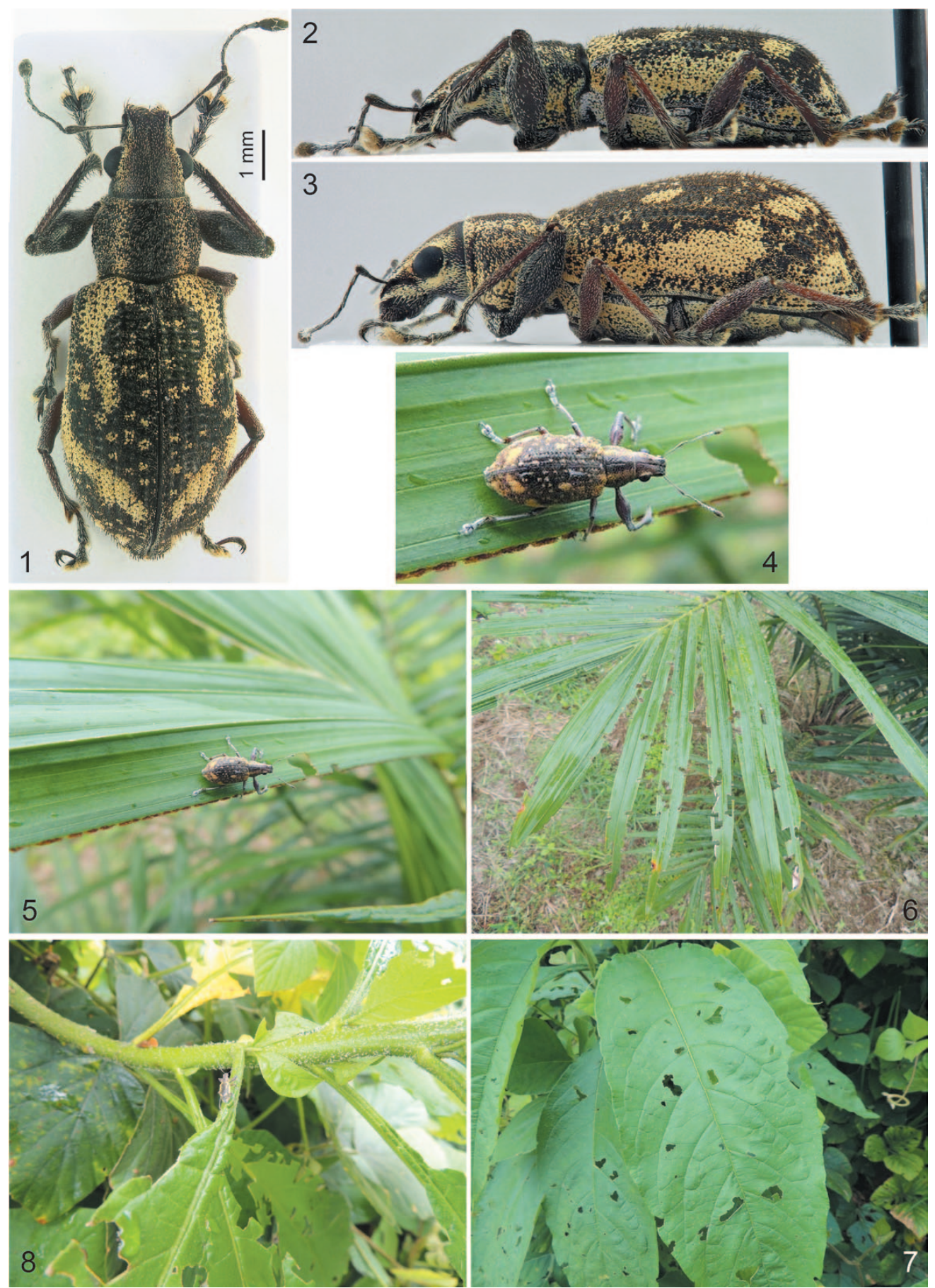

Fig. 1-8. - Galapaganus howdenae howdenae Lanteri. - 1-4, Habitus : 1, mâle en vue dorsale ; 2, mâle en vue latérale ; 3, femelle en vue latérale; 4, imago sur feuille de Palmier à huile Elaeis guineensis. - 5-8, Dégâts : 5-6, dégâts sur Palmier à huile; 7, dégâts sur une Dicotylédone indéterminée localement appelée "Cadillo"; 8, dégâts sur Pueraria phaseoloides. 
Au plus fort de la pullulation, il s'est ensuite attaqué aux feuilles basses et il consommait aussi les Graminées (Poaceae) et les plantes de couverture, dont Pueraria phaseoloides (Roxb.) Benth. (Fabaceae) (fig. 7-8), sans nécessairement initier ses attaques par les bords externes des feuilles. La population a retrouvé son niveau initial à partir de janvier 2015. Un an plus tard, l'Insecte est toujours présent, mais les populations restent faibles.

$G$. howdenae howdenae est actif aux heures fraîches de la matinée. Il se cache sur la face inférieure des feuilles et se laisse tomber au sol quand on s'approche. On l'observe le matin sur un arbuste appelé localement "Cadillo" (fig. 7). Au fur et à mesure que la température augmente au cours de la journée, il se rencontre plutôt près du sol dans la couverture végétale basse. Aucun parasitoïde ni entomopathogène n'a été observé jusqu'à présent sur ce Charançon.

\section{CONCLUSION ET PERSPECTIVES}

C'est la première mention de la présence de Galapaganus howdenae dans la province d'Esmeraldas, ce qui étend vers le nord sa répartition connue.

Ce premier signalement sur jeunes palmiers à huile montre que cette espèce est susceptible d'occasionner des défoliations affectant la photosynthèse. Les surfaces cultivées en Palmier à huile en Équateur dépassant 200000 hectares, il serait judicieux d'organiser une veille sanitaire des plantations pour vérifier si le statut de ravageur de l'espèce se confirme ou s'il ne s'agit que d'une pullulation ponctuelle. En effet, rien ne permet encore d'expliquer cette soudaine pullulation ni d'affirmer qu'elle se reproduira.

Remerciements. - Les auteurs remercient le Dr Analía Lanteri du Museo de La Plata en Argentine qui a identifié l'Insecte et nous a fourni une précieuse documentation, ainsi que le Dr Hélène Perrin du Muséum national d'Histoire naturelle, à Paris, pour ses investigations. Nous remercions également Philippe Amblard de Palmelit SAS pour la relecture du manuscrit.

\section{AUTEURS CITÉS}

Corley R. H. V. \& Tinker P. B., 2016. - The Oil Palm. Fifth Edition. Wiley Blackwell, xxxi +655 p.

Howard F. W., Moore D., Giblin-Davis R. M. \& Abad R. G., 2001. - Insects on palms. Wallingford : CABI Publishing, $400 \mathrm{p}$.

Lanteri A., 1992. - Systematics, Cladistics, and Biogeography of a new Weevil Genus, Galapaganus (Coleoptera: Curculionidae) from the Galápagos Islands, and Coast of Ecuador and Perú. Transactions of the American Entomological Society, 118 (2) : 222-267.

2004. - New taxonomic and biogeographic information on Galapaganus femoratus Species Group (Coleoptera: Curculionidae: Entiminae). Transactions of the American Entomological Society, 130 (2) : $1-16$.

LePesme P., 1947. - Les Insectes des Palmiers. Paris : Paul Lechevalier éd., 903 p.

MARIAU D., 2001. - The fauna of oil palm and coconut insect and mite pests and their natural enemies. Montpellier : Cirad éd., 249 p.

Mok H.-F., Stepien C. C., Kaczmarek M., Roque Albelo L. \& Sequeira A. S., 2014. - Genetic Status and Timing of a Weevil Introduction to Santa Cruz Island, Galapágos. Journal of Heredity, 105 (3) : 365-380.

Sequeira A. S., Lanteri A. A., Scataglini M. A., Confalonieri V. A. \& Farrell B. D., 2000. - Are flightless Galapaganus weevils older than the Galápagos islands they inhabit? Heredity, 85 : 20-29. 